\title{
Can Feedback, Cooperation, Relays and Full Duplex Operation Increase the Degrees of Freedom of Wireless Networks ?
}

\author{
Viveck R. Cadambe, Syed A. Jafar \\ Electrical Engineering and Computer Science \\ University of California Irvine, \\ Irvine, California, 92697, USA \\ Email:vcadambe@uci.edu, syed@uci.edu
}

\begin{abstract}
We consider a fully connected network with $S$ full duplex source nodes, $D$ full duplex destination nodes and $R$ relay nodes, perfect feedback to source and relay nodes, and noisy cooperation between all source, relay and destination nodes. We show that this network has $\frac{S D}{S+D-1}$ degrees of freedom if the channel gains are time-varying/frequency selective. The implication of the result is that, the techniques mentioned in the title (i.e relays etc.) can affect the capacity of a network only upto a $o(\log (S N R))$ term and therefore cannot improve the degrees of freedom of a network. Certain communication scenarios excluded by our system model where these techniques improve the degrees of freedom are also identified. Bounds on the degrees of freedom of a fully connected $K$ node network emerge as a by-product of our study.
\end{abstract}

\section{INTRODUCTION}

The degrees of freedom ${ }^{1}$ of a network approximates the capacity of a networks as

$$
C(\mathrm{SNR})=d \log (\mathrm{SNR})+o(\log (\mathrm{SNR}))
$$

where $d$ is the number of degrees of freedom of the network and $C(\mathrm{SNR})$ represents the capacity of a network as a function of the signal to noise ratio (SNR). At high SNR, the $o(\log (\mathrm{SNR}))$ term becomes negligible in comparison to $\log (\mathrm{SNR})$ and the accuracy of the approximation becomes $100 \%$. By studying wireless networks at high SNR, the degrees of freedom approach de-emphasizes noise and addresses the effects of interference on wireless networks.

The degrees of freedom approach has aided in finding a powerful tool in combating interference in the form of interference alignment [2]-[5]. Interference alignment is the idea that signals are constructed so that they cast overlapping shadows at the receivers where they constitute interference while they remain distinguishable at the receivers where they are desired. Through an interference alignment based achievable scheme, the capacity of the $K$ user interference network was characterized as $C(\mathrm{SNR})=(K / 2) \log (\mathrm{SNR})+o(\log (\mathrm{SNR}))$.

\footnotetext{
${ }^{1}$ Also referred to as multiplexing gain [1] or capacity pre-log.
}

Equivalently the interference network has $K / 2$ degrees of freedom [6].

Reference [7] generalized the interference network to the $X$ network, a network where there is an independent message from every source node to every destination node. It was shown that the $S \times D X$ network - the $X$ network with $S$ source nodes and $D$ destination nodes and $S D$ messages - has a capacity of $\frac{S D}{S+D-1} \log (\mathrm{SNR})+o(\log (\mathrm{SNR}))$, i.e. it has $\frac{S D}{S+D-1}$ degrees of freedom. However, we observe that the models of the interference and $X$ network excludes several of the standard techniques employed to improve capacity in wireless networks. For example, the model precludes cooperation, multi-hop network communication, feedback and full duplex operation. The main goal of this paper is to study the impact of relays, feedback, noisy cooperation and full-duplex operation on the capacity of wireless networks.

We generalize the $S \times D X$ network of [7] to the $S \times R \times D$ network (Figure 1). This network has $S$ fullduplex source nodes, $R$ relays, $D$ full-duplex destination nodes, perfect feedback to all source and relay nodes, noisy co-operation among all nodes. The network is assumed to be fully connected, meaning that all channel gains are non-zero. The main result of this paper is that like $S \times D X$ network, the $S \times R \times D$ network also has $\frac{S D}{S+D-1}$ degrees of freedom. While, achievability follows trivially from the interference alignment based scheme of [7], the main contribution of this paper is the converse argument presented in Theorem 1 in Section II. Therefore, in most cases, the answer to the question posed in the title of this paper is, quite surprisingly, negative. In other words, the search for improvements of the order of $\log (\mathrm{SNR})$ in most wireless networks ends in interference alignment. The techniques of relays, feedback to source/relay nodes, noisy co-operation and full-duplex operation can only improve the capacity upto a $o(\log (\mathrm{SNR}))$ term. There are, however, a few exceptions precluded by our system model where these techniques can improve the degrees of freedom. 


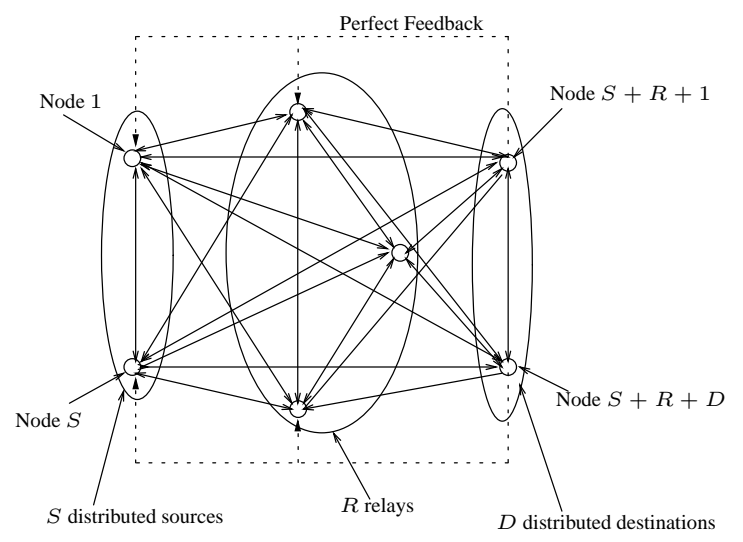

Fig. 1. The $S \times R \times D$ network

1) Relays can improve the degrees of freedom if a network is not fully connected.

2) Co-operation can increase the degrees of freedom if the cost of co-operation is not accounted for (i.e. in genie aided networks).

3) Full duplex operation can increase the degrees of freedom if source nodes can also be destination nodes for other messages.

4) Feedback can improve the degrees of freedom if it is provided to a destination node, in which case, it behaves, in effect, like an extra antenna and can be used to null out the interference.

\section{THE $S \times R \times D$ NODE $X$ NeTWORK}

Consider an $S \times R \times D$ node network, i.e., a network with $S+R+D$ nodes where nodes $1,2, \cdots, S$ are sources, nodes $S+1, S+2, \cdots, S+R$ are relays, and nodes $S+R+1, S+R+2, \cdots, S+R+D$ are destination nodes (see Figure 1). Following the definition of an $X$ network [7], for all $j \in\{1,2, \cdots, S\}$ and for all $i \in\{S+R+1, S+R+2, \cdots, S+R+D\}$, there is an independent message $W_{i, j}$ to be communicated from source node $j$ to destination node $i$.

Full duplex operation is assumed so that all nodes are capable of transmitting and receiving simultaneously. The input and output signals of the $S \times R \times D$ node network are related as:

$$
Y_{i}(n)=\sum_{j=1}^{S+R+D} H_{i, j}(n) X_{j}(n)+Z_{i}(n), n \in \mathbb{N}(1)
$$

where, at the $n^{\text {th }}$ discrete time slot, $\forall i, j \in\{1,2 \ldots S+$ $R+D\}, j \neq i, X_{j}(n)$ is the symbol transmitted by node $j, Y_{i}(n)$ is the symbol received by node $i, H_{i, j}(n)$ is the channel from node $j$ to node $i$ and $Z_{i}(n)$ is the zero mean unit variance additive white Gaussian noise (AWGN) at node $i$. We use the following notation,

$$
X_{i}^{n} \triangleq\left\{X_{i}(1), X_{i}(2), \cdots, X_{i}(n)\right\}
$$

Similar notation is used for output signals and the additive noise terms as well.

The channel coefficients $H_{i, j}(1), H_{i, j}(2), \cdots, \forall i, j \in$ $\{1,2, \cdots, S+D+R\}$ are known apriori to all nodes. We assume the network is fully connected, so that all channel coefficients can only take non-zero values. The AWGN terms $Z_{i}(n)$ have unit variance and are independent identically distributed (i.i.d.) in time and across nodes.

Perfect (noise-free) feedback of all received signals is available to all source and relay nodes, but not to the destination nodes. Therefore, for codewords spanning $N$ channel uses, the encoding functions are as follows,

$$
X_{i}(n)=\left\{\begin{array}{c}
f_{i, n}\left(W_{S+R+1, i}, \cdots, W_{S+R+D, i}, \mathbf{Y}^{n-1}\right), \\
\quad \text { if } i \in\{1,2, \cdots, S\} \\
f_{i, n}\left(\mathbf{Y}^{n-1}\right), \\
\quad \text { if } i \in\{S+1, S+2, \cdots, S+R\} \\
f_{i, n}\left(Y_{i}^{n-1}\right), \\
\quad \text { if } i \in\{S+R+1, \cdots, S+R+D\}
\end{array}\right.
$$

for $n=1,2, \cdots, N$, where

$$
\mathbf{Y}^{m}=\left(Y_{1}^{m}, Y_{2}^{m}, \ldots Y_{S+R+D}^{m}\right)
$$

The decoding functions are as follows,

$$
\begin{array}{r}
\hat{W}_{i, j}=g_{i, j}\left(Y_{i}^{N}\right), i \in\{S+R+1, \cdots, S+R+D\}, \\
j \in\{1,2, \cdots, S\}
\end{array}
$$

Note that, since a destination node does not receive feedback, it can only use its own received signal for encoding and decoding. The probability of error is the probability that there is at least one message $W_{i, j}$ that is not decoded correctly, i.e. $\hat{W}_{i, j} \neq W_{i, j}$ for some $(i, j)$. The total power across all transmitters is assumed to be $\rho$ per channel use. We denote the size of the message set by $\left|W_{i, j}(\rho)\right|$. Let $R_{i, j}(\rho)=\frac{\log \left|W_{i, j}(\rho)\right|}{N}$ denote the rate of the codeword encoding the message $W_{i, j}$, where the codewords span $N$ slots. A rate-matrix $\left[\left(R_{i, j}(\rho)\right)\right]$ is said to be achievable if messages $W_{i, j}$ can be encoded at rates $R_{i, j}(\rho)$ so that the probability of error can be made arbitrarily small simultaneously for all messages by choosing appropriately long $N$.

Let $C(\rho)$ represent capacity region of the $S \times R \times D$ node network, i.e., it represents the set of all achievable rate-matrices $\left[\left(R_{j i}(\rho)\right)\right]$. The degrees of freedom region of the $S \times R \times D$ node network is defined as

$$
\begin{aligned}
& \mathcal{D}=\left\{\left[\left(d_{i, j}\right)\right] \in \mathbb{R}_{+}^{S D}: d_{i, j}=\lim _{\rho \rightarrow \infty} \frac{R_{i, j}(\rho)}{\log (\rho)}\right. \\
& \forall(i, j) \in\{S+R+1, \ldots S+R+D\} \times\{1,2 \ldots S\}\}
\end{aligned}
$$

We present, in the theorem below, outerbounds for the degrees of freedom region and the total number of degrees of freedom of the $S \times R \times D$ network. 


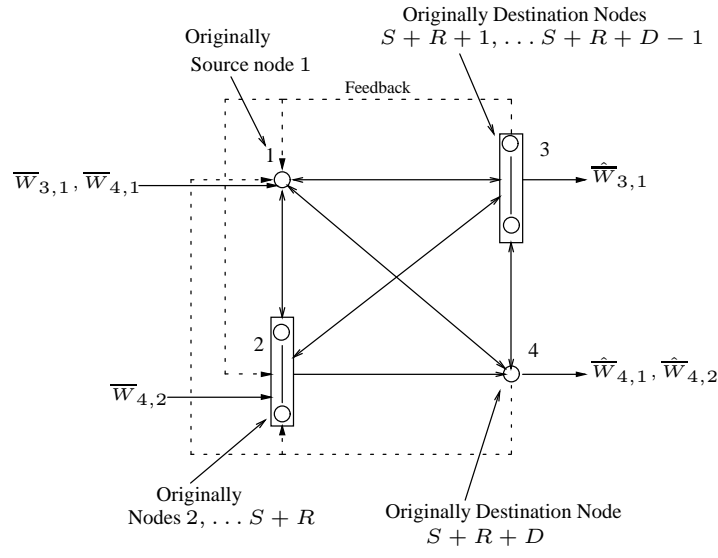

Fig. 2. Conversion of the $S \times R \times D$ network to a 4 node $X$ channel

Theorem 1: Let

$$
\begin{aligned}
& \mathcal{D}^{\text {out }} \triangleq\left\{\left[\left(d_{i j}\right)\right]:\right. \\
& \forall(u, v) \in\{1 \cdots S\} \times\{S+R+1, \cdots, S+R+D\} \\
&\left.\quad \sum_{q=S+R+1}^{S+R+D} d_{q, u}+\sum_{p=1}^{S} d_{v, p}-d_{v, u} \leq 1\right\}
\end{aligned}
$$

Then $\mathcal{D} \subseteq \mathcal{D}^{\text {out }}$ where $\mathcal{D}$ represents the degrees of freedom region of the $S \times R \times D$ node $X$ network. Furthermore,

$$
\max _{\left[\left(d_{i j}\right)\right] \in \mathcal{D}} \sum d_{i j} \leq \frac{S D}{S+D-1}
$$

Equivalently, the sum-capacity $C_{\Sigma}(\rho)$ may be expressed as

$$
C_{\Sigma}(\rho)=\frac{S D}{S+D-1} \log (\rho)+o(\log (\rho))
$$

Proof:

To prove the theorem, all we need to show is that for any $(u, v) \in\{1,2, \ldots, S\} \times\{S+R+1, S+R+$ $2, \ldots, S+R+D\}$

$$
\sum_{i=S+R+1}^{S+R+D} d_{i, u}+\sum_{j=1}^{S} d_{v, j}-d_{v, u} \leq 1
$$

For convenience, we will show the inequality for $(u, v)=(1, S+R+D)$. By symmetry, the inequality extends to all desired values of $u, v$. We therefore intend to show that

$$
\sum_{i=S+R+1}^{S+R+D} d_{i, 1}+\sum_{j=1}^{S} d_{S+R+D, j}-d_{S+R+D, 1} \leq 1
$$

To show this, we first eliminate all the messages that are not associated with either source node 1 or destination node $S+R+D$. Now, we transform the original $S \times R \times D$ node network with single antenna nodes into a $2 \times 0 \times 2$ node network, i.e., an $X$ network with 2 source nodes, zero relay nodes and 2 destination nodes where one source and one destination have multiple antennas (see Figure 2). This is done by allowing full cooperation between the $S-1$ source nodes $2, \cdots, S$ and the $R$ relay nodes $S+1, S+2, \cdots, S+R$ so that they effectively become one transmitter with $S+R-1$ antennas. Similarly, destination nodes $S+R+1, S+R+2, \cdots, S+R+D-1$ are also allowed to perfectly cooperate so that they form one receiver with $D-1$ antennas. Note that all operations described so far cannot reduce the degrees of freedom region and therefore do not contradict our outerbound argument. We represent the resulting 4 node $X$ network (Figure 2) by the following input-output equations.

$$
\bar{Y}_{i}(n)=\sum_{j=1}^{4} \bar{H}_{i, j}(n) \bar{X}_{j}(n)+\bar{Z}_{i}(n)
$$

for $i=1,2,3,4$ where $\bar{X}_{i}(n), \bar{Y}_{i}(n), \bar{Z}_{i}(n)$ represent the respectively transmitted symbol, received symbol and AWGN terms at node $i$. Note that for $i=2,3$, these terms are vectors. The definition of the channel coefficients $\bar{H}_{i, j}(n)$ is clear from the above equation and Figures 1 and 2. Multiple messages that have the same source and the same destination are combined in the 4 node $X$ network as follows:

$$
\begin{aligned}
& \bar{W}_{3,1}=\left[\begin{array}{llll}
W_{S+R+1,1} & W_{S+R+2,1} & \cdots & W_{S+R+D-1,1}
\end{array}\right] \\
& \bar{W}_{4,2}=\left[\begin{array}{llll}
W_{S+R+D, 2} & W_{S+R+D, 3} & \cdots & W_{S+R+D, S}
\end{array}\right] \\
& \bar{W}_{3,2}=\phi, \quad \bar{W}_{4,1}=W_{S+R+D, 1}
\end{aligned}
$$

Now, we have converted the $S \times R \times D$ network to a 4 node $X$ network of Figure 2 . We now intend to bound the number of degrees of freedom of this $X$ network by 1 . The bound would then imply that that the sum of the degrees of freedom corresponding all messages associated with node 1 and node $S+R+D$ in the $S \times R \times D$ network is equal to 1 as desired. Let

$$
\begin{aligned}
\bar{U}_{i}(n) & =\bar{H}_{i, 1}(n) \bar{X}_{1}(n)+\bar{Z}_{i}(n), \quad i=1,2,3,4 \\
\widetilde{U}_{1}(n) & =\left(\bar{U}_{1}(n), \bar{U}_{2}(n), \bar{U}_{3}(n), \bar{U}_{4}(n)\right) \\
\widetilde{U}_{1}^{N} & =\left(\bar{U}_{1}^{N}, \bar{U}_{2}^{N}, \bar{U}_{3}^{N}, \bar{U}_{4}^{N}\right) \\
\widetilde{U}_{2}(n) & =\left(\bar{U}_{1}(n), \bar{U}_{2}(n), \bar{U}_{3}(n)\right) \\
\widetilde{W}_{1} & =\left(\bar{W}_{4,1}, \bar{W}_{4,2}\right) \\
\widetilde{W}_{2} & =\left(\bar{W}_{3,1}, \bar{W}_{4,1}, \bar{W}_{4,2}\right)
\end{aligned}
$$

Then, it can be shown that statements $S_{1}(n), S_{2}(n), S_{3}(n)$ described below are true

$$
\begin{array}{lcl}
S_{1}(n): & \bar{X}_{1}^{n} & \leftarrow \widetilde{W}_{2}, \widetilde{U}_{1}^{n-1} \\
S_{2}(n): & \bar{X}_{2}^{n}, \bar{X}_{3}^{n}, \bar{X}_{4}^{n} & \leftarrow \bar{W}_{4,2}, \widetilde{U}_{1}^{n-1} \\
S_{3}(n): & \bar{Y}_{1}^{n}, \bar{Y}_{2}^{n}, \bar{Y}_{3}^{n}, \bar{Y}_{4}^{n} & \leftarrow \bar{W}_{4,2}, \widetilde{U}_{1}^{n}
\end{array}
$$

where $A \leftarrow B$ means that the value of $A$ is completely determined by the knowledge of the value taken by $B$. The proof is in the extended paper [8]. Let a genie 
provide the messages $\bar{W}_{4,1}, \bar{W}_{4,2}$ and $\widetilde{U}_{1}^{N}$ to node 3 . Note that statement $S_{3}(n)$ implies that the node can construct $\bar{Y}_{i}(n), i=1,2,3,4$ using this side information from the genie. Using Fano's inequality, we can write

$$
\begin{aligned}
& N \bar{R}_{3,1}(\rho)-N \epsilon \\
& \leq I\left(\bar{W}_{3,1} ; \bar{W}_{4,1}, \bar{W}_{4,2}, \widetilde{U}_{1}^{N}\right) \\
& =H\left(\widetilde{U}_{1}^{N} \mid \widetilde{W}_{1}\right)-H\left(\widetilde{U}_{1}^{N} \mid \widetilde{W}_{2}\right) \\
& =\sum_{n=1}^{N} H\left(\widetilde{U}_{1}(n) \mid \widetilde{W}_{1}, \widetilde{U}_{1}^{n-1}\right)-\sum_{n=1}^{N} H\left(\widetilde{U}_{1}(n) \mid \widetilde{W}_{2}, \widetilde{U}_{1}^{n-1}\right) \\
& \stackrel{(a)}{=} \sum_{n=1}^{N} H\left(\bar{U}_{4}(n) \mid \widetilde{W}_{1}, \widetilde{U}_{1}^{n-1}\right) \\
& +\sum_{n=1}^{N} H\left(\widetilde{U}_{2}(n) \mid \widetilde{W}_{1}, \widetilde{U}_{1}^{n-1}, \bar{U}_{4}(n)\right) \\
& -\sum_{n=1}^{N} H\left(\widetilde{U}_{1}(n) \mid \widetilde{W}_{2}, \widetilde{U}_{1}^{n-1}, \bar{X}_{1}(n)\right) \\
& \stackrel{(b)}{\leq} \sum_{n=1}^{N} H\left(\bar{Y}_{4}(n) \mid \widetilde{W}_{1}, \widetilde{U}_{1}^{n-1}, \bar{Y}_{4}^{n-1}\right) \\
& +\sum_{n=1}^{N} H\left(\widetilde{U}_{2}(n) \mid \bar{U}_{4}(n)\right) \\
& -\sum_{n=1}^{N} H\left(\bar{Z}_{1}(n), \bar{Z}_{2}(n), \bar{Z}_{3}(n), \bar{Z}_{4}(n)\right) \\
& \quad \stackrel{(c)}{\leq} \sum_{n=1}^{N} H\left(\bar{Y}_{4}(n) \mid \bar{Y}_{4}^{n-1}, \widetilde{W}_{1}\right)+o(\log (\rho)) \\
& \therefore \bar{R}_{3,1}(\rho) \leq \frac{1}{N} H\left(\bar{Y}_{4}^{N} \mid \widetilde{W}_{1}\right)+o(\log (\rho))
\end{aligned}
$$

where the simplification in the third summand in the RHS in (a) and first summand in the RHS in (b) result because of $S_{1}(n), S_{2}(n), S_{3}(n)$. Note that we have also used the fact that conditioning reduces entropy in (b) and in (c). The second summand in (b) can be shown to be $o(\log (\rho))$ using the fact that Gaussian variables maximize conditional entropy (See extended paper [8] for details). Now, using Fano's inequality to bound rates of messages intended for node 4 , we can write

$$
\begin{aligned}
\bar{R}_{4,1}(\rho)+\bar{R}_{4,2}(\rho) \leq & \frac{1}{N} I\left(\bar{W}_{4,1}, \bar{W}_{4,2} ; \bar{Y}_{4}^{N}\right) \\
= & \log (\rho)+o(\log (\rho)) \\
& -\frac{1}{N} H\left(\bar{Y}_{4}^{N} \mid \widetilde{W}_{1}\right)
\end{aligned}
$$

where the final inequality can be derived from the standard upper-bound on entropy using Gaussian variables. Adding up the upperbounds on $\bar{R}_{3,1}(\rho)$ and $\bar{R}_{4,1}(\rho)+$ $\bar{R}_{4,2}(\rho)$

$$
\bar{R}_{3,1}(\rho)+\bar{R}_{4,1}(\rho)+\bar{R}_{4,2}(\rho) \leq \log (\rho)+o(\log (\rho))
$$

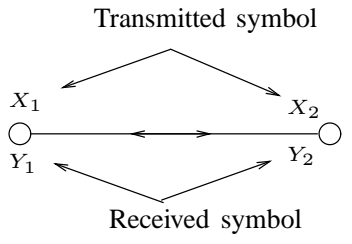

(a)

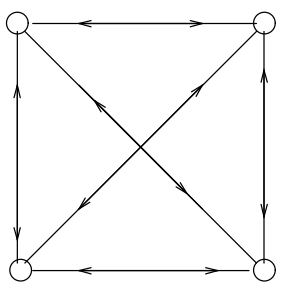

(b)
Fig. 3. $K$ user full duplex network with (a) $K=2$, (b) $K=4$

Thus, the total number of degrees of freedom of the 4 node $X$ network described is upper-bounded by 1 . This implies that

$$
\sum_{i=S+1+R}^{S+R+D} d_{i, 1}+\sum_{j=1}^{S} d_{S+R+D, j}-d_{S+R+D, 1} \leq 1
$$

The desired bound on the degrees of freedom region automatically follows. Summing all equations of the above form provides the desired bound on the total number of degrees of freedom.

\section{III. $K$ USER Full Duplex Network}

In this section, we present bounds on the degrees of freedom of the $K$ user full duplex network (see Figure 3 ). Consider a fully connected network with $K$ full duplex nodes $1,2, \ldots K$. There exists a message from every node to every other node in the network so that there are a total of $K(K-1)$ messages in the system. The message from node $j$ to node $i$ is denoted by $W_{j, i}$. Let $H_{i, j}(n)$ represent the channel gain between nodes $i$ and $j$ corresponding to the $n$th symbol. The channel gains satisfy $H_{i, j}(n)=H_{j, i}(n)$ and $H_{i, i}=0$. As usual, all nodes have apriori knowledge of all channel gains. The input-output relations in this channel are represented by

$$
Y_{i}(n)=\sum_{j=1}^{K} H_{i, j}(n) X_{j}(n)+Z_{i}(n),
$$

where $Y_{i}(n), X_{i}(n), Z_{i}(n), i=1,2 \ldots K$ represent respectively, the received symbol, the transmitted symbol and the AWGN term at node $i$. For codewords spanning $N$ symbols, we assume that the transmitted codeword $X_{i}(n)$ depends on messages $W_{j, i}, j=1,2 \ldots i-$ $1, i+1, \ldots K$, and received symbols $Y_{i}^{n-1}$. Similarly, we assume that the decoded message at each node $i$ is a function of received symbols $Y_{i}^{N}$ and messages $W_{j, i}, j=1,2 \ldots i-1, i+1, \ldots K$. Note that we do not allow feedback in this system. The rate and degrees of freedom region is defined similar to the $S \times R \times D$ network. 


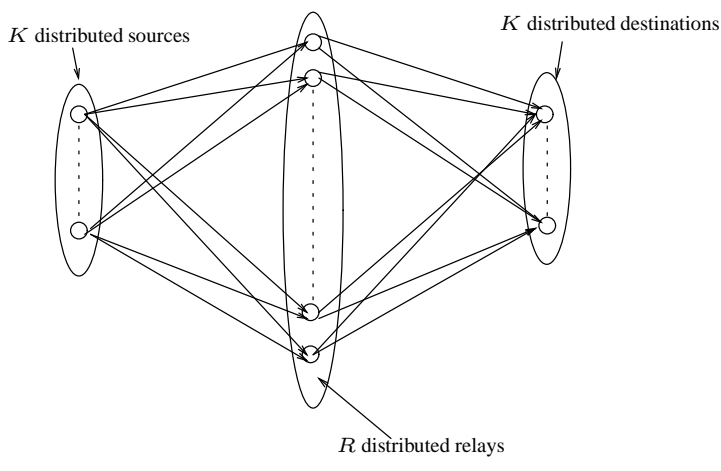

Fig. 4. The parallel relay channel

Theorem 2: The sum capacity $C_{f d}(\rho)$ of the $K$ user full duplex network as a function of power $\rho$ satisfies the following bounds

$$
\begin{aligned}
& C_{f d}(\rho) \geq \frac{K(K-1)}{2 K-2} \log (\rho)+o(\log (\rho)) \\
& C_{f d}(\rho) \leq \frac{K(K-1)}{2 K-3} \log (\rho)+o(\log (\rho))
\end{aligned}
$$

Equivalently, the number of degrees of freedom $d_{f d}$ of the network maybe bounded as

$$
\frac{K(K-1)}{2 K-2} \leq d_{f d} \leq \frac{K(K-1)}{2 K-3}
$$

The reader is referred to the extended paper [8] for a proof.

\section{Discussion of Results}

In this section, we discuss certain communication scenarios precluded by the model of the $S \times R \times D$ network where the techniques mentioned in the title improve the degrees of freedom performance.

The parallel relay network [9] with $K$ source and destination nodes and $R$ relays (Figure 4) presents an interesting example where the impact of Theorem 1 can be observed. Like the $K$ user interference channel, there are $K$ messages in this network - one message from a source node to its corresponding destination node. Reference [7] show that if $R \gg K$, the network has approximately $K$ degrees of freedom if the relays are full-duplex nodes. However, the network considered in [7] is not fully connected since the links from source nodes to destination nodes are absent. Theorem 1 implies that if the network were fully connected with direct links from source to destination nodes, then the network has $K / 2$ degrees of freedom. Therefore, in the fully connected case, by artificially imposing the half-duplex constraint on the relays, the relay aided scheme of [7] (and [9]) can only provide an alternative achievable scheme to the usual interference alignment based scheme which achieves $K / 2$ degrees of freedom.

The fact that full duplex operation improves the degrees of freedom of a network follows from the result of Theorem 2. For example, consider a network with $K$ nodes. If the nodes were half-duplex, then half the nodes behave as transmitters and half behave as receivers to form a $K / 2$ user $X$ channel. This channel has $d_{h d}=\frac{K^{2}}{4 K-4}$ degrees of freedom. From the bounds in Theorem 2 , it can easily be verified that $d_{f d} \geq d_{h d}$. The implication of Theorem 1 is that full-duplex operation can only improve degrees of freedom of a network if the source nodes can also be the destinations. Similarly, while noisy co-operation and feedback to source nodes cannot improve the degrees of freedom performance, improvements can be observed in networks with genie aided co-operation and feedback to decoding nodes (See [2], [8], [10] and references therein for examples)

\section{CONCLUSION}

We show that relays, perfect feedback to source nodes, full duplex operation and noisy co-operation can affect the capacity of a fully-connected wireless network only upto an $o(\log (\mathrm{SNR}))$ term. In such networks, the search for capacity improvements of the order of $\log (\mathrm{SNR})$ ends in interference alignment. We also note that there are certain exceptions precluded by our model, where these techniques can improve the degrees of freedom. Our study provides insight into the type of networks which are likely to derive maximum benefit from the techniques mentioned, especially at high SNR.

\section{REFERENCES}

[1] L. Zheng and D. N. Tse, "Packing spheres in the grassmann manifold: A geometric approach to the non-coherent multiantenna channel," IEEE Trans. Inform. Theory, vol. 48, Feb 2002.

[2] S. A. Jafar and S. Shamai, "Degrees of freedom region of the mimo x channel," Information Theory, IEEE Transactions on, vol. 54, no. 1, pp. 151-170, Jan. 2008.

[3] M. A. Maddah-Ali, A. S. Motahari, and A. K. Khandani, "Signaling over mimo multi-base systems: Combination of multiaccess and broadcast schemes," Information Theory, 2006 IEEE International Symposium on, July 2006.

[4] M. A. Maddah-Ali, A. S. Motahari, and A. K. Khandani, "Communication over x channel: Signalling and multiplexing gain," Technical Report: UW-ECE-2006-12, University of Waterloo, July 2006.

[5] M. A. Maddah-Ali, A. S. Motahari, and A. K. Khandani, "Communication over x channel: Signalling and performance analysis," Technical Report: UW-ECE-2006-27, University of Waterloo, December 2006.

[6] V. R. Cadambe and S. A. Jafar, "Interference alignment and degrees of freedom region for the $\mathrm{k}$ user interference channel," arxiv.org, 2007. arxiv eprint $=\mathrm{cs} / 0707.0323$.

[7] V. R. Cadambe and S. A. Jafar, "Degrees of freedom of wireless x networks," arxiv.org, 2007. arxiv eprint $=\mathrm{cs} / 0711.2824$.

[8] V. R. Cadambe and S. A. Jafar, "Capacity of wireless networks within o( $\log (\mathrm{snr}))$ - the impact of relays, feedback, cooperation and full-duplex operation," arxiv.org, Feb 2008. eprint - arxiv:0802.0534.

[9] H. Bölcskei, R. U. Nabar, O. Oyman, and A. J. Paulraj, "Capacity scaling laws in MIMO relay networks," IEEE Transactions on Wireless Communications, vol. 5, pp. 1433-1444, June 2006.

[10] N. Devroye and M. Sharif, "The multiplexing gain of mimo x-channels with partial transmit side information," Information Theory, 2007 IEEE International Symposium on, July 2007. 